\section{Comparative analysis of different hydrogels for the bioprinting of 3D in vitro skeletal muscle models}

\author{
Flaminia Aliberti,1,2 \\ Lorenza Rinvenuto, ${ }^{2}$ Giada Loi, ${ }^{3}$ \\ Laura Benedetti,2 Franca Scocozza, 3 \\ Flavio Lorenzo Ronzoni, ${ }^{4}$ Maurilio \\ Sampaolesi, ${ }^{2}$ Gabriella Cusella, ${ }^{2}$ \\ Michele Conti, ${ }^{3}$ Gabriele Ceccarelli ${ }^{2}$ \\ 1Center for Inherited Cardiovascular \\ Diseases, Transplant Research Area, \\ Fondazione IRCCS Policlinico San \\ Matteo, Pavia; ${ }^{2}$ Department of Public \\ Health, Experimental Medicine and \\ Forensic, Center for Health Technologies \\ (C.H.T.)-Human Anatomy Unit, \\ University of Pavia, Pavia; ${ }^{3}$ Department \\ of Civil Engineering, University of \\ Pavia, Pavia; ${ }^{4}$ Department of \\ Biomedical Sciences, Humanitas \\ University, Rozzano, Italy
}

\begin{abstract}
In this study we demonstrated an application of 3D Bioprinting using different commercially available hydrogels (CELLINK AB, Sweden) with the aim to identify the most suitable biomaterial for the proliferation and differentiation of murine muscle cells $(\mathrm{C} 2 \mathrm{C} 12)$.
\end{abstract}

\section{Introduction}

Despite the developments and innovations introduced in the biomedical field, nowadays, the replacement of tissues or organs, irreparably damaged by injury or disease, is an unsolved problem. ${ }^{1}$ For this reason, great expectations have been emerged from Regenerative Medicine and Tissue Engineering fields, which aim to repair, regenerate, and reproduce irreversibly damaged tissues and organs using viable cells which confer specific human tissue-specialized properties to artificial materials. ${ }^{2}$ As a case study, hereby, we analysed at different time points, the proliferation and differentiation capabilities of C2C12 cells in different commercial bioinks and encapsulated in a fibrinogen based-bioink co-printed on a PCL scaffold, using cells seeded on plastic as controls. ${ }^{2}$ Moreover, the expression patterns of master regulatory myogenic genes and cell viability have been analysed in order to evaluate the capabilities of the scaffolds to induce myoblast elongation, alignment and differentiation. ${ }^{3}$

\section{Materials and Methods}

Preparation of Bioinks: Commercially available hydrogels were provided by CELLINK (CELLINK ${ }^{\circledR}$ FIBRIN, CELLINK ${ }^{\circledR} \quad$ GelMA A， CELLINK ${ }^{\circledR}$ GelXA CELLINK AB, Gothenburg, Sweden) and applied following the manufacturer protocol.

3D bioprinting: C2C12 myoblast laden into the hydrogels were printed using CELLINK INKREDIBLE+ (Cellink AB, Gothenburg, Sweden), a pneumatic extrusion-based 3D bio-plotter. To ensure printing process sterility the INKREDIBLE+ was placed under sterile hood and UV light was turned on before printing. The bio-ink cartridge was connected to a $0,41 \mathrm{~mm}$ nozzle diameter and to the air pressure. Printing pressure and speed were set at $13 \mathrm{kPa}$ and $600 \mathrm{~mm} / \mathrm{min}$, respectively.

C2C12 myoblast cell culture: C2C12 myoblast cells were cultured in Dulbecco's Modified Eagle's Medium (DMEM; SigmaAldrich, St.Louis, MO) containing 1\% antibiotic and $10 \%$ fetal bovine serum (FBS; Gemini Bio-Products, Sacramento, CA, USA), and incubated in $5 \% \mathrm{CO}_{2}$ at $37^{\circ} \mathrm{C}$. The medium was changed every second day. For inducing differentiation, myoblasts were cultured in DMEM with $2 \% \mathrm{FBS}$.

Live/Dead assay: After 24h, 7, 14, 21 and 28 days of cell culture on a hierarchical scaffold, the myoblast cells were stained using $0.15 \mathrm{mM}$ calcein AM and $2 \mathrm{mM}$ ethidium homodimer-1 for $40 \mathrm{~min}$ in dark room. Live and dead cells were analyzed by images of stained samples captured by fluorescence microscopy (Vico Confocale, Nikon, Japan). The live cells were indicated by their green colour, and dead cells were indicated by their red colour.

RNA extraction: Total RNA extraction was carried out using the reagents provided by the Direct-zol RNA Miniprep kit, applying the protocol suggested by the manufacturer Zymo Research. NanoDrop ${ }^{\mathrm{TM}}$ (Thermo-Fisher Scientific) was used to quantify the concentration of the extracted RNA.

Gene expression analysis: The extracted RNA was quantified by Nanodrop ${ }^{\mathrm{TM}}$, retrotranscribed into cDNA using the iScript ${ }^{\mathrm{TM}}$ cDNA Synthesis Kit (Biorad, CA, USA) and subsequently analysed by qRT-PCR. The expression of the master regulatory myogenic genes involved in muscle differentiation was evaluated.
Correspondence: Flaminia Aliberti, Center for Inherited Cardiovascular Diseases, Transplant Research Area, Fondazione IRCCS Policlinico San Matteo, Pavia; Department of Public Health, Experimental Medicine and Forensic, Center for Health Technologies (C.H.T.)-Human Anatomy Unit, University of Pavia, Pavia, Italy.

E-mail: flaminia.aliberti01@universitadipavia.it

Key words: 3D bioprinting; tissue engineering; hydrogels; regenerative medicine; $\mathrm{C} 2 \mathrm{C} 12$ myoblast cell line.

Acknowledgments: This work has been supported by grants from CARIPLO 20150634 . Commercially available hydrogels were kindly provided by CELLINK (CELLINK AB, Gothenburg, Sweden).

Disclosures: The authors declare no conflict of interests.

Conference presentation: This paper was presented at the Third Centro 3R Annual Meeting - L'era delle 3R: modelli in silico, in vitro e in vivo per promuovere la ricerca traslazionale 30 September - 1 October 2021, Evento online organizzato dal Politecnico di Torino.

Received for publication: 9 July 2021.

Accepted for publication: 7 September 2021.

This work is licensed under a Creative Commons Attribution NonCommercial 4.0 License (CC BY-NC 4.0).

CC Copyright: the Author(s), 2021

Licensee PAGEPress, Italy

Biomedical Science and Engineering 2021; 4(s1):146 doi:10.4081/bse.2021.146

Immunofluorescence: Phalloidin and MF20 were used as antibodies directed towards actin and myosin the two fundamental proteins of muscle differentiation.

\section{Results}

Live/dead assay was performed to observe the viability of $\mathrm{C} 2 \mathrm{C} 12$ cells in the 3 Dprinted bio-constructs. The number of live cells was directly counted to assess the proliferation rate. As expected, based on image analysis, the average viability of cells in almost all structures was $90 \%$ within $24 \mathrm{~h}$ after printing which significantly increased in both fibrinogen-based hydrogel, fibrinogen hydrogel and PCL after 7, 14 and 21 days, indeed, a very small number of dead cells were observed after seven days. This suggests that commercial bio-inks and PCL 
as scaffold are biocompatible and allows C2C12 survival and proliferation. To quantify gene expression levels for the myogenesis (e.g., MyoD, Myf5 and MCK) we used real time PCR. Preliminary molecular biology results indicated that, at 7 and 14 days of culture, muscle genes are more expressed in control cells with respect to cells growth in the bio-inks and in the bio-ink co-printed with PCL. At 21 and 28 days $\mathrm{C} 2 \mathrm{C} 12$ cells bioprinted in fibrinogen-based hydrogel expressed in a statistically significance muscle genes, indicating the beginning of the differentiation process.

\section{Discussion and Conclusions}

In this study, a commercial cell-laden bio-inks and PCL as scaffold were applied to enable skeletal muscle differentiation of C2C12 murine myoblasts. We observed that the myoblasts grew properly into the printed fibrinogen material, with an excellent viability and with a good level of myotubes formation. Furthermore, we have demonstrated a positive effect of PCL and Fibrin hydrogel co-printing that allows us to mimic the structural properties of native tissue, in particular topographical cues to guide cell orientation. Further experiments are needed in order to ameliorate the geometry of the PCL and to design a device capable of inducing mechanical stimulation to promote the development of organized myotubes.

\section{References}

1. Medicina Rigenerativa. Patron Editore; 2009.

2. Gungor-Ozkerim PS, Inci I, Zhang YS, et al. Bioinks for 3D bioprinting: an overview. Biomater Sci 2018;6:915946.

3. Sharples AP, Al-Shanti N, Stewart CE. $\mathrm{C} 2$ and $\mathrm{C} 2 \mathrm{C} 12$ murine skeletal myoblast models of atrophic and hypertrophic potential: relevance to disease and ageing? J Cell Physiol 2010;225: 240-50. 